\title{
Sexually transmitted diseases: Impact of molecular laboratory diagnostic methods on disease control and prevention
}

\author{
Rosanna W Peeling PhD, Lai King Ng PhD
}

I

$\mathrm{n}$ recent years, reportable sexually transmitted diseases (STDs) in Canada have been on a steady decline as a result of effective provincial STD control programs. Although STDs in aggregate still represent the most frequently reported communicable diseases in Canada, the trends in declining disease incidence for bacterial STDs are encouraging $(1,2)$ (Figure 1). In 1997, there were only 76 cases of primary and secondary syphilis (incidence rate, 0.3/100,000 population) reported in Canada, with four provinces and two territories reporting no infectious syphilis cases (Figure 2 ). Over the past 10 years, the incidence of gonorrhea in Canada has decreased from a reported rate of $219.8 / 100,000$ population in 1980 to $14.9 / 100,000$ population in 1997. Elimination of endemic gonorrhea and syphilis in Canada may now be feasible.

Genital chlamydial infections became nationally reportable in 1990 and have emerged as the most frequently reported communicable disease in Canada, with 49,173 reported cases in 1991. Over $70 \%$ of genital chlamydial infections are asymptomatic. Women with undetected and untreated chlamydial infections are at risk of developing long term reproductive sequelae of pelvic inflammatory disease, ectopic pregnancy and tubal infertility. Chlamydia control programs, including the screening of asymptomatic infection in women, were initiated in many provinces in the mid- to late-1980s because the cost of pelvic inflammatory disease and its sequelae of ectopic pregnancy and infer- tility, largely due to chlamydial and gonococcal infections, was estimated at more than \$2 million/week in Canada (3). Steady improvements in laboratory diagnostic tests and the availability of single dose oral treatment have led to an average rate of decline in the reported rates of chlamydial infection of 7.7\%/year between 1991 and 1996. However, the rate of decline decreased to $1.8 \%$ in 1997 (2). The national rate of genital chlamydial infections now (as of 1999) stands at $112.7 / 100,000$ population, seven times the national rate for gonorrhea. The change in rate of decline may be complex and may reflect a transition to a different phase of the STD epidemic in Canada (4).

At low disease prevalence, different STDs may reside in subpopulations with different epidemiological characteristics and sexual network dynamics. Molecular tools developed for research and diagnosis of STDs will become increasingly important for disease control and prevention (5). Molecular typing methods can be used to construct accurate sexual networks and improve our understanding of disease transmission patterns within the population. Nucleic acid-based amplification tests (NAATs) such as the polymerase chain reaction (PCR) and ligase chain reaction offer improved sensitivity for the screening and diagnosis of chlamydial and gonococcal infections. The sensitivity of NAATs allows the use of noninvasive specimens such as urine specimens. Screening for chlamydial and gonococcal infections can now be carried out in nontraditional settings

National Laboratory for Sexually Transmitted Diseases, Bureau of Microbiology, Laboratory Centre for Disease Control, Health Canada, Winnipeg, Manitoba

Correspondence: Dr Rosanna W Peeling, Laboratory Centre for Disease Control, 1015 Arlington Street, Winnipeg, Manitoba R3E 3R2.

Telephone 204-789-2144,fax 204-789-2140,e-mail rosanna_peeling@hc-sc.gc.ca

This work has been previously published in Can J Infect Dis 1999;10:387-90. 


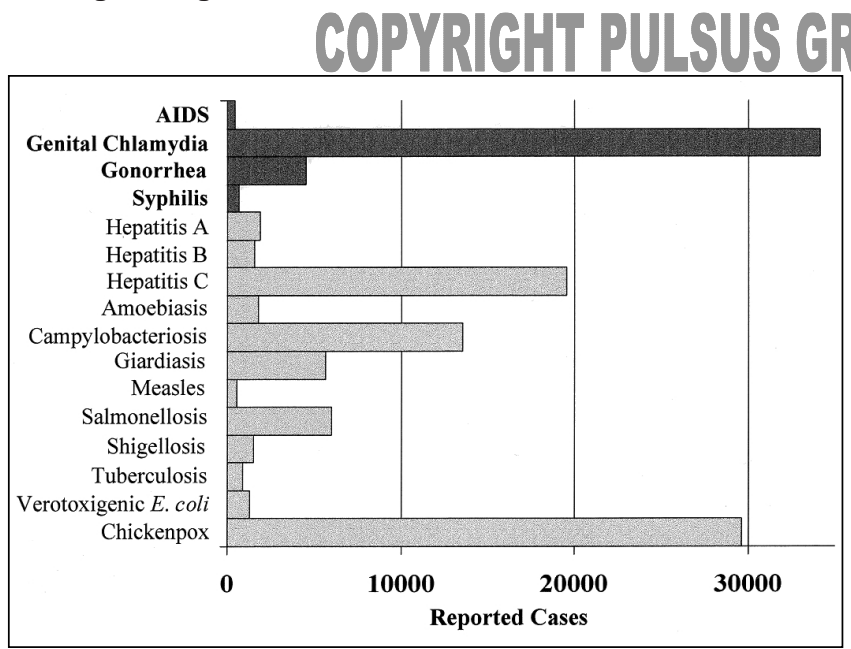

Figure 1) Reported number of cases of selected notifiable communicable diseases in Canada in 1997. E coli Escherichia coli. Data compiled from reference 1

where at-risk individuals can be targeted, irrespective of their health-seeking behaviours. As the paradigm for the laboratory diagnosis for STDs gradually shifts from biological to molecular amplification, it is important that we assess the impact of molecular methods on STD control and prevention. Safeguards must be put in place to prevent potential loss of capacity to monitor antibiotic resistance and to determine the biological characteristics of these pathogens in relation to virulence.

\section{GONORRHEA}

Although culture is the recommended method for the laboratory diagnosis of Neisseria gonorrhoeae in Canada, the organism often does not survive the transport conditions from many remote areas of Canada to the diagnostic laboratory (6). Hence, nonculture tests such as enzyme immunoassays have been in use in remote areas of Canada for many years. The sensitivity and specificity of these antigen detection tests are less than desirable, but offer a viable alternative to culture because specimen viability is not required. Antigen detection tests are also less expensive than culture because specimens can be batched for testing, and testing is automated. In recent years, nucleic acid-based tests, with or without amplification, have become commercially available for the laboratory diagnosis of $\mathrm{N}$ gonorrhoeae in Canada. These tests not only offer superior test performance compared with antigen detection tests, but also additional advantages in terms of specimen collection and processing such as the use of noninvasive specimens and the detection of multiple pathogens using a single specimen in a single amplification reaction $(7,8)$. Nucleic acid-based tests are more costly than antigen detection tests and require more technical expertise to avoid false positive results due to specimen contamination and false negative results due to the inhibition of the amplification reaction. However, the improved sensitivity, the convenience of duplex testing of gonococcal and chlamydial infections, and the ease of specimen collection and transport favour the switch to molecular testing if resources are available.

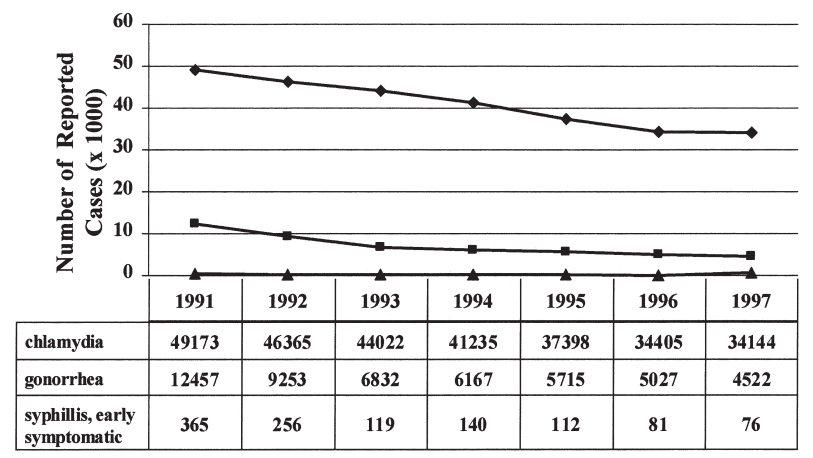

Figure 2) Trends in sexually transmitted diseases, 1991 to 1997. Graph denotes the number of cases reported by year. Chlamydia, - Gonorrhea, $\Delta$ Early symptomatic syphilis. Data from reference 2

A recent survey by Health Canada's National Laboratory for STDs in Winnipeg showed that while all of the 15 public health and hospital laboratories surveyed are currently offering culture for gonorrhea, $27 \%$ are concurrently using nucleic acid-based techniques for laboratory diagnosis (9). Because the number of specimens submitted for gonococcal testing is low, some laboratories have opted to use the chlamydia-gonorrheda duplex tests. This is not only a cost-saving measure, but, in fact, may also be a practical alternative because technologists may lose technical competency for performing culture and identification when it is rarely needed. The gradual loss of our capacity to culture the gonococci in Canada may have a negative impact on disease control in terms of surveillance for antibiotic resistance and biological characterization of isolates with different disease manifestations. Currently, the recommended treatment for gonococcal infections is cefixime (Suprax, Rhône-Poulenc Rorer, Canada) with ceftriaxone (Rocephin, Hoffmann-La Roche Limited, Canada) and ciprofloxacin (Cipro, Bayer Inc, Canada) or ofloxacin as alternative regimens (6). Increased ciprofloxacin and cephalosporin susceptibility has already been observed in Canada (unpublished data, National Laboratory for STDs). In 1997, 2.8\% of 566 isolates with any antimicrobial resistance from the provinces were resistant to ciprofloxacin. Ongoing monitoring activity for resistance to different antibiotics is, therefore, an important priority. The present gonococcal antimicrobial resistance surveillance system is based entirely on biological and not molecular methods of determining antimicrobial susceptibility patterns. The establishment and maintenance of a national network to maintain laboratory expertise and capacity in Canada for the culture and performance of antimicrobial susceptibility monitoring for $N$ gonorrhoeae is urgently needed.

The National Laboratory for STDs conducted a study in 1991 and 1992 to standardize methods for gonococcal antimicrobial susceptibility testing in Canada as part of a Pan American Health Organization initiative for monitoring of antibiotic resistance in the Americas. Currently, the 


\section{PEESONAR USE ONLY DO NON COPY

National Laboratory for STDs provides quality assurance for laboratory methods of antimicrobial susceptibility testing to public health laboratories. Until molecular methods for identifying resistance markers are developed, resources should be allocated toward the maintenance of these culture-based networks in collaboration with the provincial departments of health.

The gonococci as a pathogen have traditionally been characterized using auxotyping and serotyping of biological isolates. Molecular typing schemes have been developed, but consensus on a universal typing scheme has not been reached (10). Molecular typing methods can also be used to construct accurate sexual networks and improve the understanding of disease transmission patterns within a population. As part of the national goals strategy, molecular typing methods are currently being developed by the National Laboratory for STDs in collaboration with various laboratories in Canada and other international agencies. These methods will be an important component of disease control strategies for mapping sexual networks to delineate disease transmission patterns and to discriminate between endemic and imported strains.

\section{SYPHILIS}

The incidence of syphilis is now at an all time low in Canada (Figure 2). The resurgence of syphilis in the United States has not manifested itself in Canada (11). Nevertheless, there have been outbreaks of syphilis in Canada in high risk populations characterized by low socioeconomic status, intravenous drug use, commercial sex and, in some cases, positive HIV status (9). Although various types of diagnostic methods have been developed for the laboratory diagnosis of syphilis, serology remains the method of choice. Serology is inexpensive, rapid and easy to perform. Despite the low disease incidence and low demand for service, many laboratories in Canada continue to offer syphilis serology. The National Laboratory for Bacteriology at Laboratory Centre for Disease Control in Winnipeg provides quality assurance of syphilis testing by sending out proficiency panels to public health laboratories across Canada four times a year.

There are a number of areas where molecular testing may improve the sensitivity of laboratory diagnosis for syphilis (12,13). A multiplex PCR test, developed for the detection of Treponema pallidum, herpes simplex virus and Haemophilis ducreyi, has improved the sensitivity and accuracy of diagnosis of genital ulcers (14). DNA-based tests will also improve the diagnosis of neurosyphilis by detecting $T$ pallidum in cerebrospinal fluid, although data on the role of these tests in the management of neurosyphilis are needed. The laboratory diagnosis of syphilis in HIV patients is problematic because of low antibody responses. Molecular tests may offer an alternative to visualizing the pathogen by darkfield microscopy or immunofluorescence. Diagnostic laboratories in Canada do not use molecular tests at present.
National and international initiatives for syphilis elimination are being planned. The prospect of elimination of endemic syphilis is favourable because the treponeme has a long incubation period of between 10 and 90 days and its growth rate is slow. Thus, there is ample time to identify sexual contacts and treat them to interrupt the chain of transmission. To understand the patterns and dynamic of disease transmission, molecular typing methods are being developed (15). These typing schemes will be useful in the construction of accurate spread networks for the sexual transmission of disease and will be important tools for outbreak investigation and control.

\section{GENITAL CHLAMYDIAL INFECTION}

The hallmark of chlamydial infection is the long duration of infectiousness and its asymptomatic nature in the majority of infected individuals. The use of laboratory tests for the screening of asymptomatic individuals within a population is, therefore, an important component of an effective control program to eliminate the reservoir of infection in that population. Antigen detection tests such as enzyme immunoassays have been shown to have limited value as screening tests because of their low sensitivity, especially for asymptomatic men. NAATs provide improved sensitivity with the result that noninvasive specimens, such as urine or vaginal swabs and secretions, can be used for chlamydia testing and screening. This is important for chlamydia control programs in two ways. First, chlamydia control programs in the past have been hampered by the lack of access to youth and young adults. Approximately $70 \%$ of reported genital chlamydial infections in Canada are in the 15- to 24-year age group (9). Young women infected with chlamydia are especially at risk for developing long term reproductive complications resulting in chronic pelvic pain, ectopic pregnancy or tubal infertility. Yet youth and young adults traditionally are less likely to access the health care system than older adults. The use of noninvasive specimens can, therefore, facilitate the design of innovative approaches to screening outside of the traditional clinic-based setting. Second, the ratio of reported infections in men to women is 1:6 in the 15- to 19-year age group. This mainly reflects the reluctance of asymptomatic men to come in for screening or testing. They are, however, more likely to agree to the collection of a urine specimen rather than a urethral swab for screening.

In a recent STD laboratory services survey by the National Laboratory for STDs, over $60 \%$ of 15 major public health laboratories in Canada offer nucleic acid-based testing for genital chlamydial infections. Twenty per cent of laboratories offer culture in addition to other nonculture tests, mainly for medicolegal specimens. Although there have only been sporadic reports of antimicrobial resistance for chlamydia, there is increasing interest in developing molecular methods for monitoring resistance. The maintenance of culture capacity and expertise will be required to validate these molecular methods. 


\section{COPYRIGHT PULSUS GROUP INC, = DO NOT COPY}

The rate of decline of reported rates of chlamydial infection decreased from $7.7 \%$ to $1.8 \%$ in 1997 (2). This sudden drop in decline rates may reflect more testing and better case-finding because of increasing use of NAATs, but it may also signal the transition to a new phase of the disease epidemic (4). In Manitoba, chlamydial infection, in response to control programs carried out in the past decade, is now concentrated in urban core groups characterized by low socioeconomic status and high rates of partner change (16). Instead of the traditional risk factors for screening, such as young age, it will be important to develop new screening algorithms using NAATs. Molecular methods for genotyping chlamydia have been used as tools for mapping the spread and maintenance networks in these high risk populations for formulating strategies for disease control (9).

\section{FUTURE CHALLENGES}

The National Laboratory for STDs moved from Ottawa to Winnipeg in the summer of 1998. The new laboratory in Winnipeg now includes a section of viral STDs, which performs human papillomavirus detection and typing, as well as herpes simplex virus serology and detection by PCR. Because there are no national data on the prevalence of viral STDs, plans are under discussion with national and provincial partners for studies to determine disease burden for viral STDs. The National Laboratory for STDs remains committed to reference services for bacterial STDs, such as the evaluation of new diagnostic tests and the provision of quality assurance programs, as well as the maintenance of laboratory surveillance databases for the collection and dissemination of national trends to the stakeholders. The laboratory, in collaboration with the Division of STD Prevention and Control in the Bureau of HIV/AIDS, STD and TB at the Laboratory Centre for Disease Control, and the provincial and territorial department of health, is working toward a national goals strategy for the endemic elimination of syphilis and gonorrhea, and reduction of the incidence of genital chlamydial infections by $50 \%$ by the year 2010 (9). The development of molecular methods for typing syphilis and gonococcal strains is in progress. For chlamydial infections, the incidence of disease in adolescents and other at-risk populations is still unacceptably high. The use of molecular methods to design innovative strategies to access high risk populations and reduce the reservoir of infection in the population is a priority. With effective implementation of these STD control strategies, a reduction in the development of long term reproductive sequelae of genital chlamydial and gonococcal infections is anticipated.

\section{CONCLUSIONS}

As STDs enter the declining phase of the epidemic, different STDs may reside in subpopulations with different epidemiological characteristics and sexual network dynamics. Disease control strategies now need to be tailored to the unique epidemiology of each STD. Culture methods need to be maintained for monitoring antimicrobial resistance and for validating molecular methods to detect antibiotic resistance. Molecular tools offer improved diagnostic test performance, and high resolution molecular technology will be useful for discriminating between strains of different origin. This knowledge can be applied to outbreak investigations and control, as well as the mapping of sexual networks to further our understanding of disease transmission patterns and dynamics. Molecular laboratory methods will be an important component of STD control and prevention strategies in the next decade.

ACKNOWLEDGEMENTS: The authors gratefully acknowledge the assistance of Chris Sheardown in the preparation of this manuscript.

\section{REFERENCES}

1. Health Canada. Notifiable Diseases Summary. Can Commun Dis Rep 1999;25-20.

2. Health Canada. Laboratory Centre for Disease Control. Bureau of HIV/AIDS, STD and TB Update Series. STD Epi Updates. Ottawa: Health Canada, 1999.

3. Todd MJ, Estany A, Mclaren R. Cost of pelvic inflammatory disease and associated sequelae in Canada. Can Dis Wkly Rep 1988;14:206-8.

4. Wasserheit JN, Aral SO. The dynamic topology of sexually transmitted disease epidemics: implications for prevention strategies. J Infect Dis 1996;174(Suppl 2):S201-13.

5. Peeling RW, Mabey DCW, Holmes KK. Molecular techniques for HIV and STDs: Implications for research and disease control in the new millenium. In: Peeling RW, Sparling PF, eds. Sexually Transmitted Diseases: Methods and Protocols. Totowa: Humana Press, 1999:219-31.

6. Laboratory Centre for Disease Control Expert Working Group on Canadian Guidelines for Sexually Transmitted Diseases. Canadian STD Guidelines, 1998 edn. Ottawa: Minister of Public Works and Government Services Canada, 1998.

7. Gaydos CA, Quinn TC. Neisseria gonorrhoeae: Detection and typing by probe hybridization, PCR and LCR. In: Peeling RW, Sparling PF, eds. Sexually Transmitted Diseases: Methods and Protocols. Totowa: Humana Press, 1999:219-31.

8. Farrel DJ. Evaluation of Amplicor Neisseria gonorrhoeae PCR using cppB nested PCR and 16S rRNA PCR. J Clin Microbiol 1999;37:386-90.

9. Health Canada. Division of STD Prevention, Bureau of HIV/AIDS, STD and TB, Laboratory Centre for Disease Control. Proceedings of the STD Research Forum and National Goals Strategy Meeting, Ottawa, February 25 to 26, 1999.

10. Hesse S, Low N, Molokwu B, et al. Molecular typing as an epidemiological tool in the study of sexual networks. Lancet 1995;346:976-7.

11. Dunn RA, Rolfs RT. The resurgence of syphilis in the United States. Curr Opin Infect Dis 1991;4:3-11.

12. Jethwa HS, Schmitz JL, Dallabetta G, et al. Comparison of molecular and microscopic techniques for detection of Treponema pallidum in genital ulcers. J Clin Microbiol 1995;33:180-3.

13. Centurion-Lara A, Castro C, Shaffer JM, van Voorhis WC, Marra CM, Lukehart SA. Detection of Treponema pallidum by a sensitive reverse transcriptase PCR. J Clin Microbiol 1997;35:1348-52.

14. Orle KA, Gates CA, Martin DH, Body BA, Weiss JB. Simultaneous PCR detection of Haemophilus ducreyi, Treponema pallidum, and herpes simplex virus types 1 and 2 from genital ulcers. J Clin Microbiol 1996;34:49-54.

15. Pillay A, Liu H, Chen CY, et al. Molecular subtyping of Treponema pallidum subspecies pallidum. Sex Trans Dis 1998;25:408-14.

16. Blanchard JF, Moses S, Greenaway C, Orr P, Hammond GW, Brunham RC. The evolving epidemiology of chlamydial and gonococcal infections in response to control programs in Winnipeg, Manitoba, Canada. Am J Public Health 1998;88:1496-502. 